\title{
RF Self-Interference Cancellation Using Phase Modulation and Optical Sideband Filtering
}

\author{
Xiuyou Han, Member, IEEE, Bofan Huo, Yuchen Shao, Chao Wang, and Mingshan Zhao
}

\begin{abstract}
A novel optical approach to implement RF self-interference cancellation for full-duplex communication using phase modulation and optical sideband filtering is proposed and demonstrated experimentally. Based on the inherent out-of-phase property between the left and right sidebands of phase-modulated signal and optical sideband filtering, the RF self-interference cancellation is achieved by tuning the delay time and amplitude in the optical domain. RF self-interference cancellation for single frequency and microwave with various bandwidths of $1 \mathrm{MHz}$, $5 \mathrm{MHz}$ and $10 \mathrm{MHz}$ is experimentally demonstrated to verify the proposed technique.
\end{abstract}

Index Terms- RF self-interference cancellation, phase modulation, optical sideband filtering

\section{INTRODUCTION}

$\mathrm{C}$ Tompared with the conventional Time Division Duplex (TDD) and Frequency Division Duplex (FDD), the Full-Duplex scheme transmits and receives signals simultaneously in the same frequency band, which can significantly improve the throughput and the spectrum efficiency and is considered as a candidate technology for the fifth generation (5G) wireless communication [1], [2]. However, in the Full-Duplex scheme, due to the collocation of the transmitting and receiving antennas, the high power transmitted signal will interfere with the in-band weak received signal, which is called self-interference or co-site interference [2]. The interference cannot be simply removed by a notch filter or a narrow bandpass filter because the same frequency band is used for both transmitter and receiver. Therefore, more efficient methods to remove the self-interference for real application of the Full-Duplex scheme are highly desired. In the past few years electronic interference cancellation methods were developed to remove the interference signal from the received signals [3], [4]. However, this kind of method suffers from narrow bandwidth, high loss, and low precision time delay.

To overcome the limitations of electronic methods, optical self-interference cancellation (SIC) approaches with the advantages of wider operational bandwidth, higher precision,

This work was supported in part by Natural Science Foundation of Liaoning Province (201402002), International Science \& Technology Cooperation Program of China (2014DFG32590), National Pre-Research Foundation of China (6140450010305), and Fundamental Research Funds for the Central Universities (DUT15ZD231 and DUT2015TD47).

X.Y. Han, B.F. Huo, Y.C. Shao and M.S. Zhao are with the School of Physics and Optoelectronic Engineering, Dalian University of Technology, Dalian 116024, China (e-mail: xyhan@dlut.edu.cn, bfhuo1991@126.com, yuchenshao@mail.dlut.edu.cn,mszhao@dlut.edu.cn )

C. Wang is with the School of Engineering and Digital Arts, University of Kent, Canterbury CT2 7NT, U.K.( e-mail: c.wang@kent.ac.uk)

Copyright (c) 2017 IEEE and lower loss have been proposed to deal with the co-site interference [5]-[9]. In [5] two Mach-Zehnder modulators (MZMs) biased at inverted quadrature points were utilized to implement the electrical-to-optical (E/O) conversion of all the received signals, including the weak signal of interest (SOI) and the strong interference signal, and the tapped reference signal from the transmitter. After tuning the delay time and amplitude of the received signals in the optical domain by a tunable optical delay line (TODL) and a variable optical attenuator (VOA), the interference and reference signals cancelled each other out upon a photo detector due to exact out of phase and however identical magnitude, and the SOI was recovered. In [6] a single compact dual-drive Mach-Zehnder modulator (DD-MZM) with the bias voltage difference of $\mathrm{V}_{\pi}$ between the two arms was proposed to realize the $\mathrm{E} / \mathrm{O}$ conversion. By aligning the time delay and amplitude of the interference and cancellation signals in the electronic domain, the SOI was successfully recovered. The bias voltage for E/O modulators in [5] and [6] should be controlled accurately in order to maintain the exact out-of-phase condition, which increases the complexity of the SIC systems. In [7] two phase modulators combined with two photo detectors were utilized to implement the $\mathrm{E} / \mathrm{O}$ and $\mathrm{O} / \mathrm{E}$ conversions. The alignment of the time delay and amplitude of the interference and reference signals was realized by an electronic variable attenuator and adjustable true time delay unit. In [8] hybrid electrical and optical techniques for SIC were presented with two electro-absorption modulated lasers serving as the light source and the E/O converter simultaneously. A RF Balun transformer was always needed to invert the phase of the tapped signal from the transmitter. Therefore the electronic devices used in [7] and [8] may limit the operating frequency range of the SIC systems. In [9] an integratable dual-parallel polarization modulator (DP-PolM) based RF SIC method was presented, where optical power control was achieved by tuning the polarization state of the input optical signal into the DP-PolM, avoiding the need of a VOA. However, the polarization state may be affected by the environmental conditions and the system stability degrades.

To overcome the challenges in previous systems, in this paper, we propose and demonstrate experimentally a novel approach for optical RF SIC using phase modulation and optical sideband filtering. The left sideband of the received RF phase-modulated signal and the right sideband of tapped RF phase-modulated signal are filtered out by a single optical filter. Due to the inherent out-of-phase property between the right and left sidebands of the filtered phase-modulated signals, the RF SIC is realized during the $\mathrm{O} / \mathrm{E}$ conversion upon the PD by tuning the amplitude and delay time of the phase-modulated 
signal in the optical domain. Avoiding the complicated bias voltage control as used in intensity modulation [5], [6], the proposed scheme is greatly simplified. In addition the operational frequency range can be increased with the alignment of amplitude and delay time in the optical domain.

\section{OPERATION PRINCIPLE}

The schematic of the proposed optical RF SIC system is shown in Fig. 1. Two lasers with different wavelengths of $\lambda_{1}$ and $\lambda_{2}$ are sent to two phase modulators $\mathrm{PM}_{1}$ and $\mathrm{PM}_{2}$, respectively. The received RF signal $\mathrm{s}(t)+\mathrm{i}(t)$ from the receiver antenna, where $\mathrm{s}(t)$ is signal of interest and $\mathrm{i}(t)$ is interference signal, is modulated on the light wave of $\lambda_{1}$ via $\mathrm{PM}_{1}$. The tapped RF signal $\mathrm{r}(t)$ from the transmitter (Tx), as the reference signal, is modulated on the light wave of $\lambda_{2}$ via $\mathrm{PM}_{2}$. Through a VOA and a TODL, the phase-modulated signal in the lower path is combined with the phase-modulated signal in the upper path via a $3 \mathrm{~dB}$ optical coupler (OC) and fed to an optical filter (OF). After being filtered by the OF, the optical signals are input to the photo detector (PD).

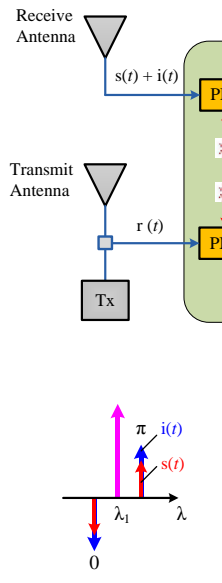

(A)

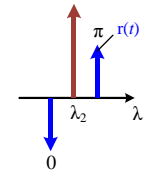

(B)
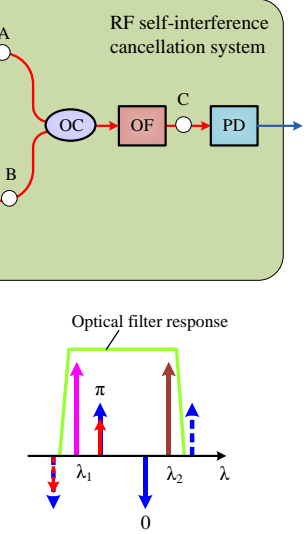

(C)

Fig. 1. The schematic of the proposed optical RF SIC system. PM, phase modulator; VOA, variable optical attenuator; TODL, tunable optical delay line; OC, optical coupler; OF, optical filter; PD, photodetector; Tx, transmitter. The insets show the spectra of the phase-modulated optical signals before (A and B) and after $(\mathrm{C})$ the $\mathrm{OF}$.

Due to the inherent property of phase modulation [10], the left and right sidebands of phase-modulated signal are out of phase as shown the spectra at point A and B in Fig. 1. The phase-modulated optical signals in the upper path and the lower path before the OF can be expressed as

$$
\begin{aligned}
& E_{\text {upper }}=E_{1} e^{\left[\mathrm{j} 2 \pi f_{C_{1} 1}+j \frac{V_{i} \cos \left(2 \pi f_{R F} t\right)}{V_{\pi 1}}\right]} \\
& E_{\text {lower }}=E_{2} \sqrt{\alpha} e^{\left[\mathrm{j} 2 \pi f_{C 2}\left(t^{\prime}+\tau\right)+j \frac{V_{r} \cos \left[2 \pi f_{R F}\left(t^{\prime}+\tau\right)\right]}{V_{\pi 2}}\right]}
\end{aligned}
$$

where $E_{1}, E_{2}, f_{\mathrm{C} 1}$ and $f_{\mathrm{C} 2}$ are the amplitude and frequency of the light waves from the two lasers; $\alpha$ is the power attenuation coefficient of the VOA; $\tau$ is the delay time of the TODL; $V_{\pi 1}$ and $V_{\pi 2}$ are the half-wave voltage of $\mathrm{PM}_{1}$ and $\mathrm{PM}_{2}$, respectively; $V_{\mathrm{i}}$ and $V_{\mathrm{r}}$ are the amplitude of the interference signal and reference signal; $f_{\mathrm{RF}}$ is the frequency of the RF signal. For simplicity, the signal of interest is not included in Eq. (1), which doesn't affect the validity of the deduction. By using the
Jacobi-Anger expansions, Eqs. (1) and (2) can be expressed as

$$
\begin{aligned}
& E_{\text {upper }}=E_{1}\left\{\begin{array}{l}
J_{1}\left(m_{1}\right) e^{\mathrm{j} 2 \pi\left(f_{C_{1}-}-f_{R F}\right) t} \\
+J_{0}\left(m_{1}\right) e^{\mathrm{j} 2 \pi f_{C 1} t} \\
+J_{1}\left(m_{1}\right) e^{\mathrm{j}\left[2 \pi\left(f_{C 1}+f_{R F}\right) t+\pi\right]}
\end{array}\right\} \\
& E_{\text {lower }}=E_{2} \sqrt{\alpha}\left\{\begin{array}{l}
J_{1}\left(m_{2}\right) e^{\mathrm{j} 2 \pi\left(f_{C 2}-f_{R F}\right)\left(t^{\prime}+\tau\right)} \\
+J_{0}\left(m_{2}\right) e^{\mathrm{j} 2 \pi f_{C 2}\left(t^{\prime}+\tau\right)} \\
+J_{1}\left(m_{2}\right) e^{\mathrm{j}\left[2 \pi\left(f_{C 2}+f_{R F}\right)\left(t^{\prime}+\tau\right)+\pi\right]}
\end{array}\right\}
\end{aligned}
$$

where $J_{0}, J_{1}$ are the 0 and $1^{\text {st }}$-order Bessel function of the first kind. When deriving Eq. (3) and (4), only the 0 - and $\pm 1^{\text {st }}$-order components are considered. The left sideband of the received RF modulated optical signal in the upper path and the right sideband of the tapped RF modulated optical signal in the lower path are filtered out by the OF. As shown the spectrum at point $\mathrm{C}$ in Fig. 1, the single sideband with carrier $(\mathrm{SSB}+\mathrm{C})$ optical signals are obtained and can be expressed as

$$
\begin{gathered}
E_{\text {upper }}=E_{1}\left\{\begin{array}{l}
J_{0}\left(m_{1}\right) e^{\mathrm{j} 2 \pi f_{C_{1} t}} \\
+J_{1}\left(m_{1}\right) e^{\mathrm{j}\left[2 \pi\left(f_{\left.\left.C_{1}+f_{R F}\right) t+\pi\right]}\right]\right.}
\end{array}\right\} \\
E_{\text {lower }}=E_{2} \sqrt{\alpha}\left\{\begin{array}{l}
J_{1}\left(m_{2}\right) e^{\mathrm{j} 2 \pi\left(f_{C 2}-f_{R F}\right)\left(t^{\prime}+\tau\right)} \\
+J_{0}\left(m_{2}\right) e^{\mathrm{j} 2 \pi f_{C 2}\left(t^{\prime}+\tau\right)}
\end{array}\right\}
\end{gathered}
$$

The RF signals are recovered by the $\mathrm{O} / \mathrm{E}$ conversion from the $\mathrm{SSB}+\mathrm{C}$ optical signals upon the $\mathrm{PD}$, and can be expressed as

$$
\begin{aligned}
& I_{a c 1} \propto a c\left(\rho \cdot E_{\text {upper }} \cdot E_{\text {upper }}^{*}\right)=A_{1} \cos \left(2 \pi f_{R F} t+\pi\right) \\
& I_{a c 2} \propto a c\left(\rho \cdot E_{\text {lower }} \cdot E_{\text {lower }}^{*}\right)=A_{2} \cos \left[2 \pi f_{R F}\left(t^{\prime}+\tau\right)\right]
\end{aligned}
$$

where $A_{1}=2 \rho E_{1}^{2} J_{0}\left(\mathrm{~m}_{1}\right) J_{1}\left(m_{1}\right), A_{2}=2 \rho \alpha E_{2}^{2} J_{0}\left(\mathrm{~m}_{2}\right) J_{1}\left(m_{2}\right), \rho$ is the responsivity of the PD.

As can be seen from Eqs. (7) and (8), when $A_{1}=A_{2}, t=t^{\prime}+\tau$ are realized by properly tuning the power attenuation coefficient $\alpha$ of the VOA and the time delay $\tau$ of the TODL, the recovered reference signal $I_{\mathrm{ac} 2}$ cancels the recovered interference signal $I_{\text {ac1 }}$. The signal of interest is therefore obtained.

\section{EXPERIMENT AND RESULTS}

An experiment based on the setup shown in Fig. 2 is performed. The RF signal from a signal generator (SG1, Agilent E8257D) is used as the signal of interest $\mathrm{s}(t)$. The RF signal from a second signal generator (SG2, Agilent E8267D) is split to two parts by an electronic $3 \mathrm{~dB}$ splitter. One part is input to $\mathrm{PM}_{2}\left(\right.$ EO Space, $\left.\mathrm{V}_{\pi}=4.15 \mathrm{~V}, 10 \mathrm{GHz}\right)$ as the reference signal $\mathrm{r}(t)$ and the other part is used as the interference signal $\mathrm{i}(t)$. The RF signal $\mathrm{s}(t)$ from SG1 and the RF signal $\mathrm{i}(t)$ split from SG2 are combined as the received signals $\mathrm{s}(t)+\mathrm{i}(t)$ via an electronic $3 \mathrm{~dB}$ combiner, and are input to $\mathrm{PM}_{1}$ (EO Space, $\mathrm{V}_{\pi}=4.08 \mathrm{~V}, 10 \mathrm{GHz}$ ). A distributed feedback laser diode (DFB-LD, Emcore-1772) with a wavelength of $\lambda_{1}=1549.056$ $\mathrm{nm}$ and a output power of $13 \mathrm{dBm}$, and a tunable laser (NKT, DK-3460) with a wavelength of $\lambda_{2}=1549.521 \mathrm{~nm}$ and a output power of $13 \mathrm{dBm}$ are used as the light sources in the upper path and the lower path, respectively. Two phase modulators ( $\mathrm{PM}_{1}$ and $\mathrm{PM}_{2}$ ) implement the $\mathrm{E} / \mathrm{O}$ phase modulation of the received RF signal $\mathrm{s}(t)+\mathrm{i}(t)$ and the reference RF signal $\mathrm{r}(t)$, respectively. 
After a VOA and a TODL the phase-modulated optical signal in the lower path is combined with the phase-modulated optical signal in the upper path via a $3 \mathrm{~dB}$ optical coupler. Then the combined phase-modulated signals are input to the $\mathrm{OF}$ (Accelink, WDM) with a bandwidth of $0.33 \mathrm{~nm}$, by which the left sideband of the received RF modulated optical signal and the right sideband of the reference RF modulated optical signal are filtered out. The $\mathrm{SSB}+\mathrm{C}$ signals are fed to a PD (Miteq SCMR-10M18G). The detected RF signals are measured with an electrical spectrum analyzer (ESA, Agilent E4440A).

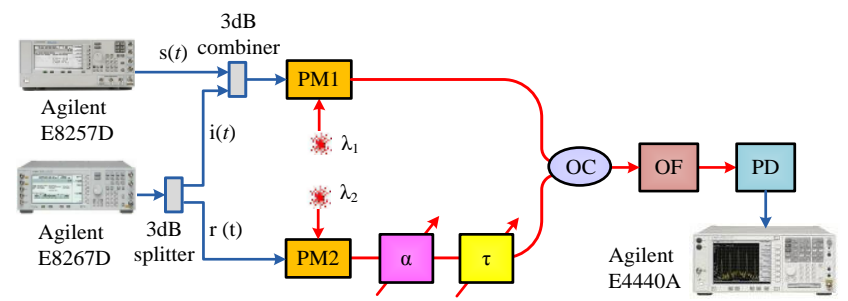

Fig. 2. The experiment setup to demonstrate the optical RF SIC.

Figure 3 show the measured optical spectra of the phase-modulated signals before and after the OF with the RF frequency of $5 \mathrm{GHz}$. Figures 3(a) and 3(b) show the optical signals modulated by the received RF signal, and Figs. 3(c) and 3(d) show the optical signals modulated by the reference RF signal. It can be seen that $\mathrm{SSB}+\mathrm{C}$ signals have been achieved by the OF. Therefore, the RF signals can be recovered by the O/E conversion from the SSB+C optical signals upon the PD.

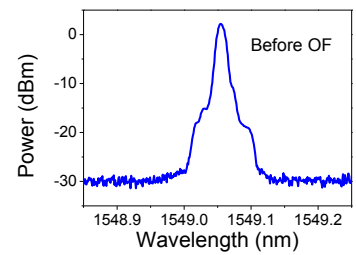

(a)

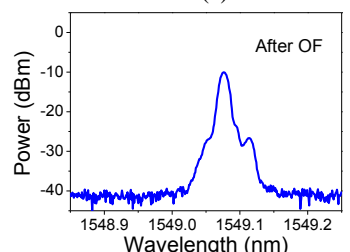

(b)

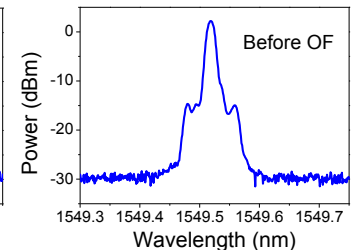

(c)

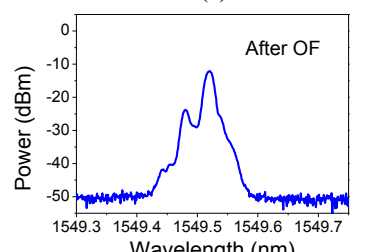

(d)
Fig. 3 The optical spectra of the phase-modulated signals $\left(f_{\mathrm{RF}}=5 \mathrm{GHz}\right)$. (a) Before and (b) after the OF with optical carrier of $\lambda_{1}$, (c) before and (d) after the $\mathrm{OF}$ with optical carrier of $\lambda_{2}$.

At first, a sinusoidal signal at $5 \mathrm{GHz}$, the standard of wireless service of WiMax (IEEE 802.16), with a power of 5 $\mathrm{dBm}$ from $\mathrm{SG} 2$ is selected to investigate the performance of single frequency SIC. Figure 4(a) shows the experimental results. By tuning off the laser source of $\lambda_{2}$, a maximum power of $-39.8 \mathrm{dBm}$ at $5 \mathrm{GHz}$ is observed on the ESA, as shown the red dash curve in Fig. 4(a). Then, by tuning on the laser source of $\lambda_{2}$, the single frequency cancellation is achieved by adjusting the VOA and TODL, with the result shown as the blue solid curve in Fig. 4(a). It can be seen that a cancellation depth of 57 $\mathrm{dB}$, which is defined as the power difference of the interference signal before and after the cancellation, is obtained. Then the frequency of the sinusoidal signal is changed to $8 \mathrm{GHz}$ with the same power level of $5 \mathrm{dBm}$ to verify the frequency extendibility of the optical SIC technique. The experimental results with and without interference cancellation are shown as the blue solid and red dash curves in Fig. 4(b), respectively. It can be seen that a cancellation depth of $56 \mathrm{~dB}$ is realized. There is about $1 \mathrm{~dB}$ difference of the cancellation depth between 5 $\mathrm{GHz}$ and $8 \mathrm{GHz}$ signals. It is due to relatively lower gain of the SIC system and the greater mismatch in the half-wave voltage of the modulators at higher frequencies.

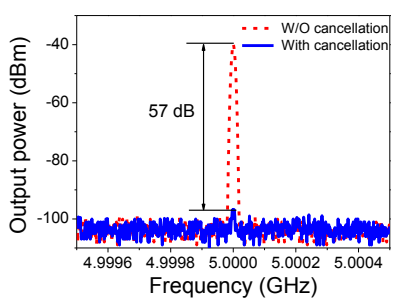

(a)

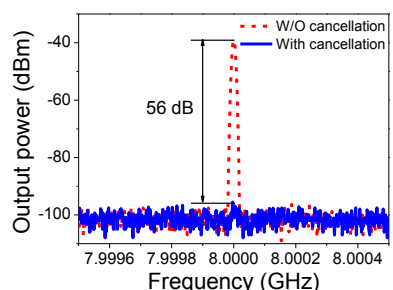

(b)
Fig. 4 The output RF spectra of single frequency signal with and without cancellation (a) $f_{\mathrm{RF}}=5 \mathrm{GHz}$ and (b) $f_{\mathrm{RF}}=8 \mathrm{GHz}$

In order to investigate the cancellation performance for RF signal with a certain bandwidth, the additive white Gaussian noise (AWGN) with a bandwidth of $1 \mathrm{MHz}$ is modulated on the RF signal from $\mathrm{SG} 2$. A $5 \mathrm{GHz}$ single tone signal with a power of $-40 \mathrm{dBm}$ from SG1 is used as the signal of interest. With the laser source of $\lambda_{2}$ being turned off, a strong interference signal is observed in the electrical spectrum as shown the red dash curve in Fig. 5 (a). Then, by tuning on the laser source of $\lambda_{2}$, the $1 \mathrm{MHz}$ bandwidth interference signal is suppressed greatly with a cancellation depth as high as $35 \mathrm{~dB}$ and the desired $5 \mathrm{GHz}$ signal is maintained, as shown the blue solid curve in Fig. 5 (a). The cancellation performance for $8 \mathrm{GHz}$ RF signal with $1 \mathrm{MHz}$ bandwidth is also investigated and the results are shown in Fig. 5(b). It can be seen from Fig. 5(b) that a cancellation depth of $34 \mathrm{~dB}$ is obtained.

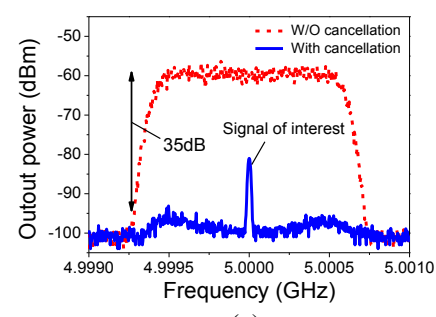

(a)

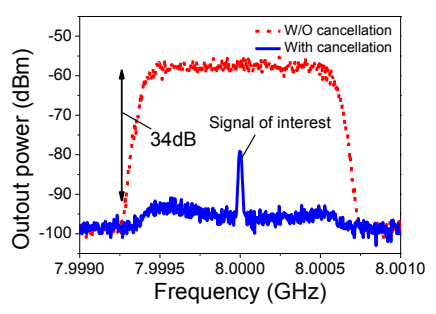

(b)
Fig. 5 The output RF spectra of an interference signal bandwidth of $1 \mathrm{MHz}$ with and without cancellation, (a) $f_{\mathrm{RF}}=5 \mathrm{GHz}$ and (b) $f_{\mathrm{RF}}=8 \mathrm{GHz}$.

Then the cancellation for RF signals with a wider bandwidth is investigated. The AWGN signal with a bandwidth of $5 \mathrm{MHz}$ is modulated on the RF signal $(5 \mathrm{dBm})$ from SG2, which is used as the interference signal. The single tone signal with a power of $-40 \mathrm{dBm}$ from SG1 is used as the signal of interest. The performance of the SIC system for $5 \mathrm{GHz}$ and $8 \mathrm{GHz}$ are measured and the results are shown in Fig. 6(a) and 6(b), respectively. It can be seen that the cancellation depth of 25.5 $\mathrm{dB}$ and $24.5 \mathrm{~dB}$ are obtained for $5 \mathrm{GHz}$ and $8 \mathrm{GHz}$ RF signals, respectively. From Fig. 5 and Fig. 6, $9.5 \mathrm{~dB}$ difference in cancellation depth is observed between RF signals with $1 \mathrm{MHz}$ and $5 \mathrm{MHz}$ bandwidth. There are mainly two reasons. One reason is that the RF power is fixed at $5 \mathrm{dBm}$ for both $1 \mathrm{MHz}$ 
and $5 \mathrm{MHz}$ bandwidth signals. The wider bandwidth is applied the smaller power is distributed at every frequency component. For the $5 \mathrm{GHz}$ RF signal as an example, the average power of about $-58.5 \mathrm{dBm}$ for $1 \mathrm{MHz}$ bandwidth but $-59.1 \mathrm{dBm}$ for 5 $\mathrm{MHz}$ bandwidth is detected without cancellation. The other reason is that the RF signal with a wider bandwidth has stronger residual noise after cancellation [11]. The maximum residual noise power for $1 \mathrm{MHz}$ bandwidth is about $-93.5 \mathrm{dBm}$, while the one for $5 \mathrm{MHz}$ bandwidth is about $-84.6 \mathrm{dBm}$.

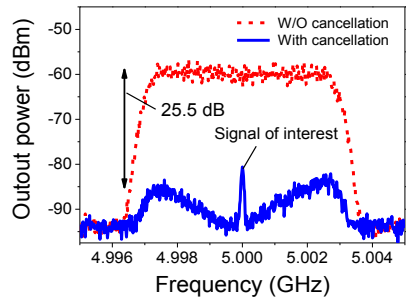

(a)

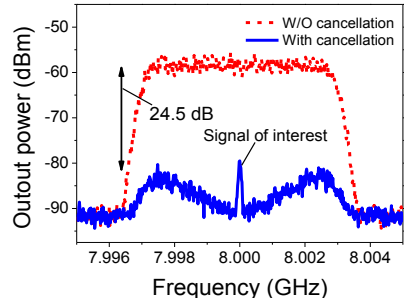

(b)
Fig. 6 The output RF spectra of an interference signal bandwidth of $5 \mathrm{MHz}$ with and without cancellation, (a) $f_{\mathrm{RF}}=5 \mathrm{GHz}$ and (b) $f_{\mathrm{RF}}=8 \mathrm{GHz}$.

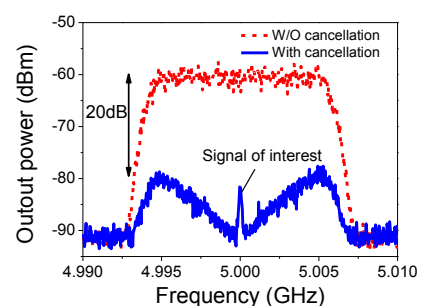

(a)

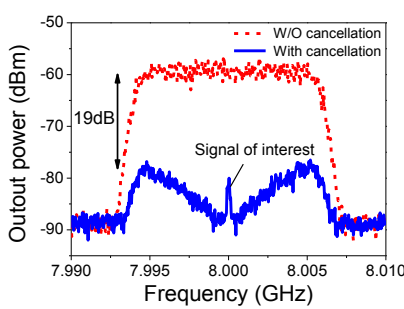

(b)
Fig. 7 The output RF spectra of an interference signal bandwidth of $10 \mathrm{MHz}$ with and without cancellation, (a) $f_{R F}=5 \mathrm{GHz}$ and (b) $f_{R F}=8 \mathrm{GHz}$.

The bandwidth of the interference signal is increased further to $10 \mathrm{MHz}$. The measured performance of the SIC system with the cancellation depth of $20 \mathrm{~dB}$ and $19 \mathrm{~dB}$ for $5 \mathrm{GHz}$ and $8 \mathrm{GHz}$ signals are shown in Figs. 7(a) and 7(b), respectively. It can be seen from Fig. 7(a) and 7(b) that the maximum residual noise $(-79.3 \mathrm{dBm} @ 5 \mathrm{GHz},-78.5 \mathrm{dBm} @ 8 \mathrm{GHz})$ in the bandwidth is higher than the recovered signal of interest (-81.6 dBm @ 5 $\mathrm{GHz},-81.8 \mathrm{dBm} @ 8 \mathrm{GHz})$. Therefore, when the RF bandwidth is increased to $10 \mathrm{MHz}$, the recovered signal of interest is degraded by the residual noise. The main factors influencing the operational bandwidth of the current experiment system include the mismatch between half-wave voltages of the two modulators [11] and the non-steep spectral edges of the OF. The operation mechanism of the latter factor can be explained as follows. Because the spectral edge of the OF is not steep enough, in order to filter out one sideband of the phase-modulated optical signal, the optical carrier and the other sideband are near the edge of the filter spectrum, which changes their initial phase relationship. The out-of-phase condition between the recovered interference and reference RF signals is not fully satisfied, leading to reduced cancellation depth. If an OF with steep edges is applied, such as the chirped sampled fiber grating [12], interference cancellation for RF signals with wide bandwidth can be achieved.

The stability of the optical SIC system with time is also investigated. The cancellation depth with time at room temperature without any protective measures (@ $5 \mathrm{GHz}$ with 5 $\mathrm{MHz}$ bandwidth) is measured. The results show that the cancellation depth remains $25 \mathrm{~dB}$ with a variation of $-0.9 \mathrm{~dB}$ to $+0.8 \mathrm{~dB}$ over one hour, indicating the good stability with time.

\section{CONCLUSION}

An optical approach for RF SIC based on phase modulation and sideband filtering is presented theoretically and experimentally. This approach utilizes the inherent out-of-phase property between the left and right sidebands of phase-modulated signals to obtain the invert phase relationship between the recovered interference and reference signals. It avoids the bias voltage control as required in intensity modulation and greatly simplifies the RF SIC system. By tuning the amplitude and time delay of the reference signal by the VOA and TODL in the optical domain, the RF SIC is realized. The performance of cancellation for RF signals with a single frequency and different bandwidth is investigated.

The preliminary experiment results verify the utility of the proposed approach. There are mainly two issues to be solved in future. One is to optimize the phase modulators and the optical filter to get the flat amplitude and linear phase response and achieve high cancellation depth over wide bandwidth. The other is to investigate the method to monitor change in amplitude and phase of the interference signal and the adaptive controlling method to tune the reference signal for the real application scenario.

\section{REFERENCES}

[1] J. I. Choi, M. Jain, K. Srinivasan, P. Levis, and S. Katti, “Achieving single channel, full duplex wireless communication," in Proc. Int. Conf. Mobile Comput. Netw., pp. 1-12. Jan. 2010.

[2] A. Sabharwal, P. Schniter, D. Guo, D. W. Bliss, S. Rangarajan, and R. Wichman, "In-band full-duplex wireless: challenges and opportunities," IEEE J. Sel. Areas Comm. Vol. 32, No. 9, pp.1637-1652, Sept. 2014.

[3] G. Boudreau, J. Panicker, N. Guo, R. Chang, N. Wang, and S. Vrzic, "Interference coordination and cancellation for $4 \mathrm{G}$ networks," IEEE Communications Magazine, Vol. 47, No. 4, pp. 74-81, Apr. 2009.

[4] S. Hong, J. Brand, J. I. Choi, M. Jain, J. Mehlman, S. Katti, and P. Levis. "Applications of self-interference cancellation in 5G and beyond," IEEE Communications Magazine, Vol. 52, No. 2, pp. 114-121, Feb. 2014.

[5] J. Suarez, K. Kravtsov, and P. R. Prucnal, "Incoherent method of optical interference cancellation for radiofrequency communications," IEEE J. Quantum Electron. Vol. 5, No.4, pp. 402-408, April 2009.

[6] Y. Zhang, S. Xiao, H. Feng, L. Zhang, Z. Zhou, and W. Hu, "Self-interference cancellation using dual-drive Mach-Zehnder modulator for in-band full-duplex radio-over-fiber system," Opt. Express, Vol.23, No.26, pp.33205-33213, Dec. 2015.

[7] E. Adles, T. Clark, M. Dennis, A. Karim, and T. McKenna, "Interferer cancellation in coherent optical RF receivers via optical phase modulation," in 25th IEEE Photonics Conference (IEEE, Burlingame, CA, 2012), pp. 218-219, Sept. 2012.

[8] Q. Zhou, H. Feng, G. Scott, and M. P. Fok, "Wideband co-site interference cancellation based on hybrid electrical and optical techniques," Opt. Lett. Vol. 39, No. 22, pp. 6537-6540, Nov. 2014.

[9] M. Huang, D. Zhu, and S. Pan, "Optical RF interference cancellation based on a dual-parallel Polarization Modulator," in Proc. Asia Commun. Photon. Conf., 2014, paper ATh1F.6

[10] H. Chi, X. Zou, and J. P. Yao, "Analytical models for phase-modulation-based microwave photonic systems with phase modulation to intensity modulation conversion using a dispersive device," J. Lightw. Technol., vol. 27, no. 5, pp. 511-521, Mar. 2009

[11] J. Suarez and P. Prucnal, "System-level performance and characterization of counter-phase optical interference cancellation," J. Lightw. Technol., vol. 28, no. 12, 1821-1831, June 2010.

[12] X.F. Chen, C.C. Fan, Y. Luo, et al., "Novel flat multichannel filter based on strongly chirped sampled fiber Bragg grating," IEEE Photonics Technol. Lett., vol. 12, no.11, pp.1501-1503, Nov. 2000. 